\title{
Developments in Psychotraumatology: A Conceptual, Biological, and Cultural Update
}

\author{
Andreas Maercker ${ }^{\mathrm{a}}$, Mareike Augsburger ${ }^{\mathrm{a}}$
}

[a] Psychopathology and Clinical Intervention, Department of Psychology, University of Zurich, Zurich, Switzerland.

Clinical Psychology in Europe, 2019, Vol. 1(1), Article e30294, https://doi.org/10.32872/cpe.v1i1.30294

Received: 2018-10-03 • Accepted: 2019-03-01 • Published (VoR): 2019-03-29

Handling Editor: Winfried Rief, Division of Clinical Psychology and Psychotherapy, Department of Psychology, Philipps-University of Marburg, Marburg, Germany

Corresponding Author: Andreas Maercker,

\section{Abstract}

Background: This report discusses recent developments of psychotraumatology mainly related to the recently published ICD-11, but also from a societal point of view.

Methods: The selected aspects of the development of this field will be presented as a scoping review.

Results: In the first section, the new concept of disorders specifically associated with stress and its relevant diagnostic groups (posttraumatic stress disorder [PTSD], complex PTSD, prolonged grief disorder, and adjustment disorder) are presented, with an emphasis on PTSD. The second section embeds these diagnostic concepts within a broader context. In particular, the concept of psychotraumatology is applied to the impact of adverse childhood experiences. More specifically, recent scientific developments are discussed with respect to biological stress research. In a third section, a global perspective is applied that reflects psychotraumatology as embedded in culturallyspecific concepts. Lastly, societal developments are taken into consideration. This section focusses on recent processes of victim acknowledgement and compensation taking place in Europe and beyond. Examples are provided for how traumatic stress is perceived and processed in society. Concepts such as continuous stress and historical trauma are also discussed.

Conclusion: Demands and opportunities of basic research and psychological interventions with a global focus are outlined.

\section{Keywords}

psychotraumatology, ICD-11, Posttraumatic Stress Disorder (PTSD), adverse childhood experiences, child abuse, acknowledgment of victims, cultural background, survivor status, compensation 


\section{Highlights}

- Psychotraumatology is an expanding field including both basic research and intervention-related research.

- Starting points of this new research area are not only potential traumatic events but also adverse childhood experiences.

- In a globalized world, cultural and societal factors play an increasingly important role in psychotraumatology.

In the early 1980s, the scientific field of psychotraumatology arose with the first description of posttraumatic stress disorder (PTSD) as a new diagnostic category in DSM-III (American Psychiatric Association, 1987). Today, this research area has been internationally recognized and well-accepted despite prevailing critical concerns and controversies (Rosen, Spitzer, \& McHugh, 2008). From its initial description in the 1980s, concepts of psychotraumatology have continuously developed. This is also reflected by the growing number of scientific publications, the founding of thematically relevant journals, as well as increasing public awareness and perception (Maercker \& Augsburger, 2017).

In the following sections, recent developments in psychotraumatology will be described. First, we will focus on new diagnostic concepts and changes in stress-related disorders associated with the launch of ICD-11. Since ICD is a major classification system used in clinical practice in many European countries, we will only briefly refer to alternative concepts as presented in DSM. A more detailed and explicit comparison of ICD-11 and DSM-5 extends beyond the scope of this review.

Second, we will describe recent developments in areas closely related to PTSD, mainly adverse childhood experiences (ACEs) and their biological impact. We focus on this specific topic for two reasons: First, in clinical practice, ACEs remain an often-neglected area despite their frequent occurrence and large burden. Second, technical advancements have resulted in a vast increase in recent years in studies focusing on biological markers associated with ACEs.

In a third section, culturally-specific models of mental disorders will be discussed with a focus on global challenges. And, lastly, aspects of public discourses are considered.

The aim of this report is to give a summarized overview of selective topics and concepts associated with recent developments in the field of psychotraumatology and in light of ICD-11. Thus, core areas were selected according to the authors' personal research foci. 


\section{New Conceptualizations of Stress-Related Disorders in ICD-11}

The updated $11^{\text {th }}$ version of the International Classification of Disorders (ICD-11) of the World Health Organization (WHO, 2018) brought about a number of significant changes in the conceptualization of stress-related mental disorders. These changes are a marked contrast to the other major classification system, the Diagnostic and Statistical Manual, version 5 (DSM-5), released by the American Psychiatric Association (APA, 2013). With ICD-11, PTSD and two additional stress-related mental disorders can now be adequately diagnosed: a complex form of PTSD and prolonged grief disorder. Moreover, a completely new symptom formulation was also grouped in this category, for adjustment disorder occurring after severe non-traumatic stressors (First, Reed, Hyman, \& Saxena, 2015; Maercker et al., 2013). Some years previously, these changes were discussed for DSM-5. But at this time the committee declared that sufficient evidence was not provided for an empirically valid distinction between PTSD and complex PTSD. As a consequence, the current PTSD diagnosis in DSM-5 also incorporates symptoms that are specified as complex PTSD in ICD-11. In addition, prolonged grief disorder cannot be diagnosed as a "full disorder" in DSM-5, but exists as a provisional diagnostic concept in the appendix (under the term "Persistent complex bereavement disorder"). Concerning adjustment disorder, the concept has remained largely the same in its transition from DSM-IV to DSM-5.

In the following sections, the four diagnoses (PTSD, complex PTSD, prolonged grief disorder, adjustment disorder) will be introduced and discussed. All criteria are based on the online version of the ICD-11 (WHO, 2018).

\section{PTSD}

PTSD manifests itself after exposure to an extremely threatening adverse event or series of events. It is characterized by the following three symptom clusters: 1) Re-experiencing of the traumatic event(s). This occurs in the form of vivid intrusive memories, such as flashbacks or nightmares. 2) Avoidance of thoughts or reminders of the traumatic event(s) or avoidance of activities, situations, or persons that elicit memories. 3) Persistent perception of heightened current threat, as characterized by an enhanced startle reaction or alertness. For a diagnosis of PTSD, all symptom clusters must persist for several weeks and lead to significant impairment in psychosocial functioning (WHO, 2018).

In contrast to both DSM-5 and ICD-10, the intrusion criterion of ICD-11 is stricter and not only requires aversive memories of the traumatic event(s), but also stronger feelings of vivid re-experiencing. In addition, the definition of hyperarousal focuses on increased perception of threat. ICD-11 prevalence rates of PTSD are lower than those for ICD-10 and are also reduced in comparison to DSM-5 (Brewin et al., 2017). Results from the World Mental Health survey indicate a lifetime prevalence from 3.0-3.4\% worldwide (Stein et al., 2014). 


\section{Complex PTSD (CPTSD)}

CPTSD can develop after exposure to an extreme and threatening event or a sequence of events, from which escape or flight is difficult or impossible. In order to give a diagnosis of CPTSD, individuals first need to fulfill all symptoms of PTSD. In addition, difficulties in three further areas must be reported: 1) Severe problems with affect regulation; 2) perception of oneself as diminished, worthless, or defective; and 3) persistent difficulties in establishing or maintaining relationships and the feeling of being close to others. As with PTSD, all symptoms need to lead to significant impairment in psychosocial functioning (WHO, 2018). This diagnosis is the successor of ICD-10 personality disorder F62.0 (Enduring Personality Change After Catastrophic Experience), but with an entirely new conceptualization. To date, limited information on prevalence rates is available for the US, Denmark, and Germany. In these countries, the rates range between $0.5-1.0 \%$, across 1-12 months (Brewin et al., 2017; Maercker, Hecker, Augsburger, \& Kliem, 2018).

\section{Prolonged Grief Disorder (PGD)}

PGD can develop after the loss of a loved one. It is marked by a persistent and intense longing for the deceased, accompanied by a strong cognitive attachment. In addition, intense emotional suffering occurs, such as sadness, feelings of guilt, anger, denial, or difficulties in accepting the death (WHO, 2018). It is important to note that all these symptoms can fall within the normal range of grieving. They may only be considered as pathological if they persistently occur over an atypically long period of time, in relation to what is considered as normative in the respective social, cultural, and religious setting. This aspect is important as it allows a broad range of culturally-related variability. For instance, in traditional Western or European cultures, symptoms that present within one year of mourning may be perceived as acceptable within this setting.

Regarding prevalence rates, studies are still lacking with respect to the new ICD-11 criteria. A recent meta-analysis on a preliminary concept of PGD reported a prevalence rate of $9.8 \%$ following the violent loss of a close person (Lundorff, Holmgren, Zachariae, Farver-Vestergaard, \& O'Connor, 2017). In general, lower rates are expected for ICD-11 (e.g., Kersting, Brahler, Glaesmer, \& Wagner, 2011).

\section{Adjustment Disorder}

Adjustment disorder manifests itself as an intense reaction towards a clearly identifiable psychosocial stressor. Typically, it occurs within one month following the starting point of the stressor. A main symptom is the cognitive attachment towards the stressor or its consequences. This can be expressed as excessive worrying, persistent burdensome thoughts about the stressor, and constant rumination. For a diagnosis of adjustment disorder, these symptoms must lead to significant impairment in psychosocial functioning. 
Improved functioning should only be possible with considerable effort. Over the course of time, a symptom remission can occur within six months (WHO, 2018). In contrast to both ICD-10 and DSM-5, this new concept of adjustment disorder brings significant changes: First, the core symptoms of preoccupation and failure to adapt are now clearly described and must be present for a diagnosis. And second, in the current formulation, the subtypes of adjustment disorder (e.g., depressive or anxious) were omitted, as previous studies showed a high degree of overlap between the subtypes (Maercker \& Lorenz, 2018). Regarding prevalence rates, a recent study reported a one-year prevalence of $2 \%$ in a representative German sample (Glaesmer, Romppel, Brahler, Hinz, \& Maercker, 2015). However, rates are much higher in risk samples. For instance, rates ranged between $13.8-17.2 \%$ in a sample of individuals who had experienced involuntary job loss in Switzerland (Perkonigg, Lorenz, \& Maercker, 2018).

The above four stress-related diagnoses not only emphasize the considerable improvements in clinical utility (Maercker et al., 2013), but also reflect the fact that therapeutic interventions for specific disorders have been developed and evaluated in recent years (Schnyder \& Cloitre, 2015). For PTSD, trauma-focused specific psychotherapeutic interventions that incorporate a variant of exposure show the best evidence for treatment efficacy (e.g., narrative exposure therapy, trauma-focused cognitive-behavioral therapy). For complex PTSD, a phase-based intervention was developed and is currently being evaluated (e.g., Cloitre, Koenen, Cohen, \& Han, 2002). For prolonged grief disorder, different treatment manuals are available (Rosner et al., 2014). Lastly, adjustment disorder is also benefitting from new interventions on a low-threshold level (Maercker, Lorenz, Perkonigg, \& Kapfhammer, 2016).

A remaining issue is the different conceptualizations of the disorders, specifically PTSD with respect to DSM-5 and ICD-11. Recent studies point to the fact that different subgroups of patients are being identified depending on the classification system used (e.g., Barbano et al., 2019). However, these discrepancies also offer the opportunity for further scientific discourse.

\section{Expanding the Concept of Psychotraumatology}

Today, it is well recognized that traumatic experiences during childhood, such as sexual abuse or physical violence, can have a long-lasting and devastating impact on later life. More recently, less severe types of traumatic experiences, such as verbal abuse, have also gained awareness as a similarly potent form of maltreatment (Teicher, Samson, Polcari, \& McGreenery, 2006). The term Adverse Childhood Experiences (ACE) incorporates a much broader range of these exposure types, including emotional or physical neglect, or peer violence. It is evident that some of these maltreatment types extend beyond the definition of a traumatic event, according to the classification of DSM or ICD. 


\section{Consequences of Adverse Childhood Experiences}

The first systematic investigation of adverse childhood experiences (the so-called ACE studies) incorporated a huge sample of 17,300 study participants and were a milestone for later research (Anda et al., 2006; Dube et al., 2001). For the first time, not only the longterm consequences of exposure to physical or sexual abuse were assessed, but also the impact of a broad range of other experiences, such as emotional abuse, physical or emotional neglect, and other risk factors in the child's environment. The ACE-studies resulted in overwhelming evidence for the significant negative impact of these experiences in later life: up to a 3.6-fold increased risk for depressive disorders, 2.4-fold increased risk for anxiety disorders, 2.7-fold increased risk for occurrence of hallucinations, 2.1-fold increase for sleeping disorders, and 7.2-fold increased risk for alcohol abuse. In addition, risk for somatic complaints was increased by 2.7 -fold, and severe obesity showed up to a 1.9-fold increased risk (Anda et al., 2006).

These ACE-studies not only led to the general acknowledgement of the detrimental effects of adverse childhood experiences, but also resulted in the development of standardized and validated measures to assess ACEs. Today, the Childhood Trauma Questionnaire is one such questionnaire investigating adverse and traumatic childhood experiences, and has thus far been used in more than 500 studies (Viola et al., 2016).

Several meta-analyses have provided further evidence and confirmed the risk for the development of mental and somatic diseases and behavioral problems as a result of adverse experiences (e.g., Augsburger, Basler, \& Maercker, in press; Hughes et al., 2017; Norman et al., 2012). Additionally, a meta-review (summarizing previous reviews) on sexual abuse, demonstrated the devastating impact of sexual abuse on later life, showing an increased risk for a broad range of severe disorders and symptoms (e.g., personality disorders, eating disorders, psychotic symptoms, sexual dysfunction, and also somatic complaints, such as pelvic pain or non-epileptic seizures); as well as impairment in social interactions, and an increased risk for future exposure to sexual violence, but also involvement in aggressive acts (Maniglio, 2009). This last aspect is particularly relevant for the field of pediatric and adolescent psychiatry (Anda et al., 2006; Augsburger, MeyerParlapanis, Bambonyé, Elbert, \& Crombach, 2015). However, the sequela of ACEs also expand to geronto-psychiatry, evident in an increased risk for cognitive deficits in older age (Burri, Maercker, Krammer, \& Simmen-Janevska, 2013).

\section{Modulation of the Biological Stress Response}

As mentioned above, adverse childhood experiences present an unspecific risk factor for increased vulnerability to later (psycho)pathology. They are assumed to have an impact on biological regulatory mechanisms in the human body. More specifically, exposure to ACEs may result in a cascade of neuro-endocrine and immunologic alterations that are associated with changes in the brain (Nemeroff, 2016; Teicher \& Samson, 2013, 2016). 
These processes refer to disturbed regulation of the human stress reaction, and the hypothalamic-pituitary-adrenal axis (HPA axis). Accordingly, structural changes are likely to occur in stress-sensitive brain regions with a high density of glucocorticoid receptors, to which the stress hormone cortisol binds (Nemeroff, 2016). Most evident is an increase in volume of the Amygdala, as well as a reduction of the Hippocampus, but structural changes in prefrontal regions have also been reported (Nemeroff, 2016; Teicher \& Samson, 2016). More recent studies investigating connectivity have demonstrated a strong connection between these brain regions. Accordingly, the inhibition of brain regions, such as the amygdala, that are involved in the processing of fear stimuli, can act in a hyperactive manner. However, different types of adverse experiences can lead to differential effects (see Norman et al., 2012; Teicher \& Samson, 2016). Similarly, brain regions are likely to have sensitive phases during a specific age period, in which they are particularly vulnerable to the effect of adverse experiences. In addition, there may be a genderspecific component. For instance, the hippocampus of girls appears to be more stress-resistant than the hippocampus of boys (Teicher \& Samson, 2016).

All these aspects can be subsumed under the term "type-and-timing" as they relate to differential effects during specific age periods and for various types of ACEs (Nemeroff, 2016; Teicher \& Samson, 2016). These new developments complement the cumulative effects of ACEs with a dose-response relationship that was reported in the initial ACEstudies (Anda et al., 2006). Teicher and Samson (2013) even argue in favor of two biologically distinct groups of patients with mental disorders that can be differentiated based on their specific neuro-biological alterations: those with exposure to ACEs and those without. This assumption has been taken up by other scientists (cf. Nemeroff, 2016) and, if proven valid, would result in huge implications for diagnostic procedures as well as the treatment of disorders.

Whilst findings of altered biological circuits offer a powerful explanation for the longterm impact of ACEs, many studies rely on cross-sectional data, thus compromising causality. However, a limited number of studies also provide evidence from a longitudinal perspective. For instance, Trickett, Noll, Susman, Shenk, and Putnam (2010) investigated long-term HPA axis activity by assessing cortisol levels in two cohorts of young women with or without exposure to sexual violence, who were followed up from a mean age of 11 until the age of 24. In accordance with previous findings, cortisol levels and trajectories between the two cohorts significantly differed. However, the sample size was rather small and potential confounders were not taken into account.

\section{Epigenetic Alterations}

The field of epigenetics investigates the direct impact of the environment on transcription of the human DNA through the process of methylation, without changing the original DNA-sequence (Marinova et al., 2017; Turecki \& Meaney, 2016). Due to its involvement in the stress reaction, focus is placed on methylation in glucocorticoid receptor 
genes (Nemeroff, 2016). Here, a large number of experimental studies with animals demonstrate increased methylation to be associated with a lack of maternal care (Nemeroff, 2016; Turecki \& Meaney, 2016). Regarding humans, similar results have been reported with respect to adverse childhood experiences (Nemeroff, 2016). A systematic review incorporating 27 studies with humans supported the assumption of increased methylation, despite different methodological approaches (Turecki \& Meaney, 2016).

Some researchers argue that alterations in methylation are not specifically induced by ACEs, but are rather a general effect associated with a broad range of mental disorders. However, previous research has provided evidence that patients with PTSD and additional exposure to ACEs showed increased rates of methylation compared to PTSD patients without exposure to ACEs (Pape \& Binder, 2014). These results are in favor of effects specifically induced by ACEs and support the previously discussed theory of a biologically distinct subtype (cf. Teicher \& Samson, 2016).

\section{Outlook on ACEs}

Overall, these findings demonstrate the future potential of research involving biomarkers and epigenetic approaches. Epigenetic processes can also aid in the identification of mechanisms involved in the trans-generational transmission of adverse experiences, as indicated by previous studies (Yehuda et al., 2016). Despite these significant findings, premature conclusions should be avoided: Many relevant studies did not incorporate potential confounding variables, thus weakening causal explanations (Nemeroff, 2016). Furthermore, the majority of studies apply cross-sectional research designs, with retrospective self-reports of ACEs (see Hughes et al., 2017). Regarding type and timing of ACEs, the heterogeneity of assessments (e.g., different scales, frequency versus severity of events) and restricted sample types further limit generalizability.

Additionally, epigenetic research itself suffers from methodological constraints: Different extraction methods (e.g., saliva versus serum), as well as non-standardized procedures for pre-processing, weaken empirical evidence. Moreover, the previously mentioned shortcomings in study designs, such as cross-sectional studies and the failure to include mediators, require a cautious interpretation of causality.

Finally, the implications of these findings for clinical practice remain less clear. Nemeroff (2016) highlights two important aspects: First, can these biological alterations be prevented by psychotherapy or pharmacotherapy, if detected early? And second, it needs to be investigated, if these biological alterations are reversible following interventions. While preliminary studies with animals and also studies with war veterans support this view, evidence is far from conclusive (Nemeroff, 2016).

In sum, the majority of findings are consistent and provide strong evidence for increased later vulnerability towards mental disorders, with relative effects for specific types of events. Further research is required in order to disentangle potential methodological constraints and draw final conclusions. 


\section{Modeling of Culturally-Specific Trauma Concepts}

Focusing on European, US-American, and Australian PTSD researchers (the so-called "Global North"), one aspect that is often neglected concerns the cultural background of patients. Thus, it is basically assumed that psychological processes and their social implications work in a universal manner across all cultures. However, both clinical practice and (cross)-cultural clinical research still have to demonstrate if these assumptions are valid (Hinton \& Good, 2016; Maercker, Heim, \& Kirmayer, 2019). This aspect is particularly relevant, as many patients with PTSD symptoms grow up in cultures other than the "Global North". Examples include individuals from war-affected regions (e.g., Afghanistan, Iraq, Syria), those who have experienced political prosecution (e.g., in the case of the Rohingya communities in Myanmar in 2017), or natural catastrophes (e.g., Banda Aceh tsunami in 2004).

Within the context of the migration and refugee movement affecting Europe in 2015, many countries began to tailor their psychological and psychotherapeutic interventions towards these groups (Silove, Ventevogel, \& Rees, 2017). However, there is still too little work on culturally-specific adaptations. This may lead to an over-simplification, which may account for the fact that many interventions developed in Western communities show less efficacy in other samples, as a recent meta-analysis indicated (Thompson, Vidgen, \& Roberts, 2018). Consequently, an extension of theoretical models is required, to help explain the development and maintenance of PTSD in a culturally-sensitive manner. Thus far, the existence of these models is rather limited (Bernardi, Engelbrecht, \& Jobson, 2018; Hinton, Ojserkis, Jalal, Peou, \& Hofmann, 2013; Maercker \& Horn, 2013). In our working group, the socio-interpersonal model of PTSD was developed, which explicitly takes cultural aspects into account (Maercker \& Hecker, 2016; Maercker \& Horn, 2013). More specifically, it works on three levels (cf. Filipp \& Aymanns, 2018):

1. The traumatized individual is an interdependent self in relation to other human beings. This stands in contrast to the independent self - a traditional differentiation in cultural psychology. It is indisputable that the self is never completely independent from its social relations, but is always interdependent, also in individualized societies from the Global North. This is mainly relevant with respect to exposure to traumatic events and the frequent arising of specific social emotions, such as guilt and shame, but also anger, rage, and thoughts of revenge. All these emotions reflect the interdependency between the self and others. Moreover, the individual's perception and self-labelling of the traumatic event and its sequela (e.g., "I am traumatized", "I am diagnosed with PTSD"), relates to the interaction with and comparison to other persons. For example, as a result of this comparison, members 
from disadvantaged communities frequently argue that their own personal experiences are not relevant as the whole community is suffering. As a consequence, they do not perceive themselves as individuals seeking help (Rechsteiner, Maercker, \& Tol, 2019).

2. The maintenance of trauma-related symptoms is embedded in a dialogical or communicative process. To date, research has mainly focused on procedures of service utilization in order to identify person-related internal and external barriers. Here, a shift is needed towards the exploration of possibilities for individuals to selfdisclose their traumatic experiences, as well as the investigation of reactions from other persons towards this disclosure (Pielmaier \& Maercker, 2011). As speechlessness and the inability to verbalize what happened is a significant facet of trauma-related disorders, this dialogue between individuals is essential. The tremendous value of relationships between two people or within a community is therefore reflected by the opportunity for individuals to overcome this speechlessness. On a more basic level, other individuals with similar experiences can additionally provide non-verbal support, resulting in feelings of emotional connection. These aspects fit well with the rationale of Narrative Exposure Therapy, which has been successfully applied in diverse international settings (cf., Schauer, Neuner, \& Elbert, 2011).

3. From a broader perspective, the societal and cultural context play a significant role in relation to the impact of traumatic experiences. Here the model becomes a sociocultural one, referring to cultural value orientation and religious or traditional cultural beliefs. For instance, cross-cultural studies indicate an association between traditional norms in the society (e.g., conformity, obedience, or benevolence) and increased rates of PTSD after exposure to interpersonal violence (Maercker et al., 2009). This leads to the essential question, with great relevance for the respective health care system, if the status of a victim or survivor is ascribed to these affected individuals in their respective society. A refusal of this societal acknowledgement of the survivor status can result in feelings of being left alone, and may lead to increased helplessness, embitterment, and fatalism. This may also contribute to a cycle of ongoing violence in fragile regions, perpetuated by inter-generational transmission (Elbert, Rockstroh, Kolassa, Schauer, \& Neuner, 2006). Inevitably, these contextual factors require a culturally-sensitive or even culturally-adapted treatment approach for patients from the "Global South" (previously termed "non-Western countries”) (Dickerson et al., 2018; Von Lersner \& Kizilhan, 2017; Whealin et al., 
2017). This cultural adaptation is visible when certain parameters are considered, such as setting, delivery mode, translation, treatment goals, local conceptualizations of disorders, the use of metaphors, and particularities of relationships (Bernal \& Sáez-Santiago, 2006). Currently, there are limited studies that consider the treatment of PTSD or complex PTSD, whilst also taking these aspects into account. However, a recent meta-analysis was published concerning E-mental health of common mental disorders and the so-called scalable psychosocial interventions. It demonstrates that treatment efficacy is linearly and positively associated with the number of culturally-adapted parameters (Harper Shehadeh, Heim, Chowdhary, Maercker, \& Albanese, 2016). Nevertheless, further research is needed, for instance, concerning culturally appropriate metaphors of adverse events (Meili, Heim, \& Maercker, 2018).

Following this socio-interpersonal model of PTSD, trauma-focused interventions also need to incorporate interventions on a group or community level (Maercker \& Hecker, 2016). This corresponds to the WHO's demand for new theoretically derived, empirically verifiable interventions for the international arena (Tol et al., 2011).

\section{Public Discussions}

Nowadays, the field of psychotraumatology is not only limited to clinical psychology and psychiatry, but extends to the overall society: It is discussed among legal experts, historians, anthropologists, politicians, the media, cultural scientists, as well as artists. In the public media, traumatic experiences and its sequela are present on a level similar to depression and substance abuse. In this section, public aspects of psychotraumatology will be discussed (cf. Maercker, 2017, p. 70 et seq.).

\section{Acknowledgement and Compensation of Survivors}

The general public has started to acknowledge the immense damage that traumatic experiences can cause to individuals' mental and physical health. This is an important step in order to remedy past failures, for instance, with respect to institutional abuse. In Germany, Austria, and Switzerland, round table discussions were initiated and commissioners were implemented for specific topics, in order to collectively process these dark chapters of the past. An example in Germany is the round table Sexual child abuse in dependent relationships and power relations in private and public institutions and in the family, and the round table Residential care in the 50s and 60s. In Austria, the position of an Independent Commissioner for Victims of the Catholic Church was implemented in 2010. Finally, in Switzerland, there is the Independent Commission of Experts on Institutional Care at the Swiss Federal Parliament. The work of these institutions is based on state-of-the-art sci- 
entific findings, which also incorporates the recent findings concerning the impact of ACEs. For instance, an investigation at the University of Ulm in Germany assessed the current health status of victims of sexual abuse, who were involved in the round table Sexual Child Abuse. They reported a high rate of mental disorders, with $40 \%$ depressive disorders, 19\% PTSD, and 18\% anxiety disorders (Spröber et al., 2014).

As previously described, it is a political and societal necessity to acknowledge the suffering of survivor groups that have been previously neglected. However, it is not appropriate to exclusively focus on the high incidence of trauma-related disorders in these groups. It is similarly important to investigate and emphasize results related to resilience, that is, reasons for overcoming traumatic stress. Increased public awareness is needed for this second aspect. Certainly, this must not imply that financial compensation is only accessible for survivors suffering from their traumatic experiences, but must be offered to all survivors. Thus, discussions about the criteria that need to be fulfilled in order to gain access to compensation need to continue (Maercker \& Augsburger, 2017).

\section{Continuous Stress and Historical Aspects of Traumatic Experiences}

In the process of re-formulating the ICD-11 grouping of disorders specifically associated with stress, a new diagnostic category was discussed: continuous trauma disorder. In many countries and regions, there is no clear onset and end of a traumatic event, but rather a constant and ongoing threat for human life (e.g. Somasundaram, 2014). Accordingly, the term "post-traumatic" is not feasible for these regions and the diagnosis of PTSD does not apply, if taken literally. From a biological viewpoint, in these circumstances the body is in a state of constant high physiological alertness in order to survive resulting in impaired body function and significant distress. Currently, best-practice suggestions are available for dealing with these aspects (World Health Organization, 2016). However, in the relevant ICD-11 working group, the incorporation of an entirely new diagnostic concept was rejected, and was instead referred to the areas of emergency psychology and medicine.

Related to this is the term "historical trauma", which describes the experiences of systematic violent discrimination, persecution, and extermination of ethnic or religious groups. It is often called "historical" if public acknowledgment is not provided and if an "atonement" is not yet sufficient (Kirmayer, Gone, \& Moses, 2014). Examples include collective traumatic experiences of the First Nations and African-Americans in the US, or the Holocaust in Europe. More recently, the term has also been used to describe nonman-made mass catastrophes, such as the tsunami in 2004, or the 2011 Fukushima nuclear disaster in Japan. Some researchers suggest that collective perceptions and pathological alterations in thoughts and behavior emerge following these events, which can be differentiated from symptoms associated with PTSD or similar diagnoses. For instance, Somasundaram (2014) reported the following changes, among others, in response to 
these experiences: general mistrust, suspicion, brutalization, a drop in morals and values, passivity, and negativism.

\section{Processing of Trauma in Other Public Areas}

Perceptions and explanations related to traumatic stress also expand beyond the previously discussed aspects and permeate into several other public areas. This is not only reflected by topics such as cultures of memory, and the occurrence of several Truth and Reconciliation Commissions following political violence; but also, art exhibitions featuring artists that try to process and integrate their biographical experiences and wounds into their artistic work. Examples for the latter include the internationally renowned conceptual artists Joseph Beuys or Marina Abramović (see Maercker, 2017). Not surprisingly, within these settings, the conceptualizations of traumatic stress and psychotraumatology can differ from a scientific point of view. Also, in this area, recent developments may not be sustainable. Nevertheless, they have the potential to aid and support individuals in overcoming their personal experiences.

Funding: The authors have no funding to report.

Competing Interests: The first author had previously chaired the work group on "Disorders specifically associated with stress" for the ICD revision at the World Health Organization from 2011-2018. However, he did not receive any reimbursement for this work. The views expressed in this article are those of the authors and do not represent the official policies or position of the WHO. The first author is member of the Editorial Board of Clinical Psychology in Europe but played no editorial role for this particular article.

Acknowledgments: The authors have no support to report.

General Note: This article is a modified and substantially extended version of an article previously published in German (Maercker \& Augsburger, 2017).

\section{References}

American Psychiatric Association. (1987). Diagnostic and statistical manual of mental disorders (3rd ed., rev.). Washington, DC, USA: Author.

American Psychiatric Association. (2013). Diagnostic and statistical manual of mental disorders (5th ed.). Arlington, VA, USA: American Psychiatric Publishing.

Anda, R. F., Felitti, V. J., Bremner, J. D., Walker, J. D., Whitfield, C., Perry, B. D., . . Giles, W. H. (2006). The enduring effects of abuse and related adverse experiences in childhood: A convergence of evidence from neurobiology and epidemiology. European Archives of Psychiatry and Clinical Neuroscience, 256(3), 174-186. https://doi.org/10.1007/s00406-005-0624-4 
Augsburger, M., Basler, K., \& Maercker, A. (in press). Is there a female cycle of violence after exposure to childhood maltreatment? A meta-analysis. Psychological Medicine.

Augsburger, M., Meyer-Parlapanis, D., Bambonyé, M., Elbert, T., \& Crombach, A. (2015). Appetitive aggression and adverse childhood experiences shape violent behavior in females formerly associated with combat. Frontiers in Psychology, 6, Article 1756.

https://doi.org/10.3389/fpsyg.2015.01756

Barbano, A. C., van der Mei, W. F., Bryant, R. A., Delahanty, D. L., deRoon-Cassini, T. A., Matsuoka, Y. J., ... Shalev, A. Y. (2019). Clinical implications of the proposed ICD-11 PTSD diagnostic criteria. Psychological Medicine, 49(3), ) 483-490. https://doi.org/10.1017/S0033291718001101

Bernal, G., \& Sáez-Santiago, E. (2006). Culturally centered psychosocial interventions. fournal of Community Psychology, 34(2), 121-132. https://doi.org/10.1002/jcop.20096

Bernardi, J., Engelbrecht, A., \& Jobson, L. (2018). The impact of culture on cognitive appraisals: Implications for the development, maintenance, and treatment of posttraumatic stress disorder. Clinical Psychologist. Advance online publication. https://doi.org/10.1111/cp.12161

Brewin, C. R., Cloitre, M., Hyland, P., Shevlin, M., Maercker, A., Bryant, R. A., . . Reed, G. M. (2017). A review of current evidence regarding the ICD-11 proposals for diagnosing PTSD and complex PTSD. Clinical Psychology Review, 58, 1-15. https://doi.org/10.1016/j.cpr.2017.09.001

Burri, A., Maercker, A., Krammer, S., \& Simmen-Janevska, K. (2013). Childhood trauma and PTSD symptoms increase the risk of cognitive impairment in a sample of former indentured child laborers in old age. PLoS One, 8(2), Article e57826. https://doi.org/10.1371/journal.pone.0057826

Cloitre, M., Koenen, K. C., Cohen, L. R., \& Han, H. (2002). Skills training in affective and interpersonal regulation followed by exposure: A phase-based treatment for PTSD related to childhood abuse. fournal of Consulting and Clinical Psychology, 70(5), 1067-1074. https://doi.org/10.1037/0022-006X.70.5.1067

Dickerson, D., Baldwin, J. A., Belcourt, A., Belone, L., Gittelsohn, J., Keawe'aimoku Kaholokula, J., ... Wallerstein, N. (2018). Encompassing cultural contexts within scientific research methodologies in the development of health promotion interventions. Prevention Science. Advance online publication. https://doi.org/10.1007/s11121-018-0926-1

Dube, S. R., Anda, R. F., Felitti, V. J., Chapman, D. P., Williamson, D. F., \& Wayne, H. G. (2001). Childhood abuse, household dysfunction, and the risk of attempted suicide throughout the life span: Findings from the adverse childhood experiences study. JAMA, 286(24), 3089-3096. https://doi.org/10.1001/jama.286.24.3089

Elbert, T., Rockstroh, B., Kolassa, I.-T., Schauer, M., \& Neuner, F. (2006). The influence of organized violence and terror on brain and mind: A co-constructive perspective. In P. Baltes, P. ReuterLorenz, \& F. Roesler (Eds.), Lifespan development and the brain: The perspective of biocultural coconstructivism (pp. 326-363). Cambridge, United Kingdom: Cambridge University Press.

Filipp, S. H., \& Aymanns, P. (2018). Kritische Lebensereignisse und Lebenskrisen: Vom Umgang mit den Schattenseiten des Lebens [Critical life events and life crises: Coping with the dark sides of life]. Stuttgart, Germany: Kohlhammer. 
First, M. B., Reed, G. M., Hyman, S. E., \& Saxena, S. (2015). The development of the ICD-11 Clinical Descriptions and Diagnostic Guidelines for Mental and Behavioural Disorders. World Psychiatry: Official fournal of the World Psychiatric Association (WPA), 14(1), 82-90. https://doi.org/10.1002/wps.20189

Glaesmer, H., Romppel, M., Brahler, E., Hinz, A., \& Maercker, A. (2015). Adjustment disorder as proposed for ICD-11: Dimensionality and symptom differentiation. Psychiatry Research, 229(3), 940-948. https://doi.org/10.1016/j.psychres.2015.07.010

Harper Shehadeh, M., Heim, E., Chowdhary, N., Maercker, A., \& Albanese, E. (2016). Cultural adaptation of minimally guided interventions for common mental disorders: A systematic review and meta-analysis. FMIR Mental Health, 3(3), Article e44. https://doi.org/10.2196/mental.5776

Hinton, D. E., \& Good, B. J. (2016). Culture and PTSD: Trauma in global and historical perspective: Philadelphia, PA, USA: University of Pennsylvania Press.

Hinton, D. E., Ojserkis, R. A., Jalal, B., Peou, S., \& Hofmann, S. G. (2013). Loving-kindness in the treatment of traumatized refugees and minority groups: A typology of mindfulness and the nodal network model of affect and affect regulation. fournal of Clinical Psychology, 69(8), 817-828. https://doi.org/10.1002/jclp.22017

Hughes, K., Bellis, M. A., Hardcastle, K. A., Sethi, D., Butchart, A., Mikton, C., . . Dunne, M. P. (2017). The effect of multiple adverse childhood experiences on health: A systematic review and meta-analysis. The Lancet Public Health, 2(8), e356-e366.

https://doi.org/10.1016/S2468-2667(17)30118-4

Kersting, A., Brahler, E., Glaesmer, H., \& Wagner, B. (2011). Prevalence of complicated grief in a representative population-based sample. fournal of Affective Disorders, 131(1-3), 339-343. https://doi.org/10.1016/j.jad.2010.11.032

Kirmayer, L. J., Gone, J. P., \& Moses, J. (2014). Rethinking historical trauma. Transcultural Psychiatry, 51(3), 299-319. https://doi.org/10.1177/1363461514536358

Lundorff, M., Holmgren, H., Zachariae, R., Farver-Vestergaard, I., \& O'Connor, M. (2017). Prevalence of prolonged grief disorder in adult bereavement: A systematic review and meta-analysis. Journal of Affective Disorders, 212, 138-149. https://doi.org/10.1016/j.jad.2017.01.030

Maercker, A. (2017). Trauma und Traumafolgestörungen. München, Germany: Beck.

Maercker, A., \& Augsburger, M. (2017). Psychotraumatologie: Differenzierung, Erweiterung und öffentlicher Diskurs [Psychotraumatology: Differentiation, extension and public discourse]. Nervenarzt, 88(9), 967-973. https://doi.org/10.1007/s00115-017-0363-6

Maercker, A., Brewin, C. R., Bryant, R. A., Cloitre, M., van Ommeren, M., Jones, L. M., . . Reed, G. M. (2013). Diagnosis and classification of disorders specifically associated with stress: Proposals for ICD-11. World Psychiatry: Official fournal of the World Psychiatric Association (WPA), 12, 198-206. https://doi.org/10.1002/wps.20057

Maercker, A., \& Hecker, T. (2016). Broadening perspectives on trauma and recovery: A sociointerpersonal view of PTSD. European fournal of Psychotraumatology, 7, Article 29303. https://doi.org/10.3402/ejpt.v7.29303 
Maercker, A., Hecker, T., Augsburger, M., \& Kliem, S. (2018). ICD-11 prevalence rates of posttraumatic stress disorder and complex posttraumatic stress disorder in a German nationwide sample. Journal of Nervous and Mental Disease, 206, 270-276. https://doi.org/10.1097/NMD.0000000000000790

Maercker, A., Heim, E., \& Kirmayer, L. J. (2019). Cultural clinical psychology and PTSD. Boston, MA, USA: Hogrefe.

Maercker, A., \& Horn, A. B. (2013). A socio-interpersonal perspective on PTSD: The case for environments and interpersonal processes. Clinical Psychology \& Psychotherapy, 20(6), 465-481. https://doi.org/10.1002/cpp.1805

Maercker, A., \& Lorenz, L. (2018). Adjustment disorder diagnosis: Improving clinical utility. The World fournal of Biological Psychiatry, 19 (Suppl. 1), S3-S13. https://doi.org/10.1080/15622975.2018.1449967

Maercker, A., Lorenz, L., Perkonigg, A., \& Kapfhammer, H. P. (2016). Anpassungsstörungen. In H.-J. Möller, G. Laux, \& H. P. Kapfhammer (Eds.), Psychiatrie, Psychosomatik, Psychotherapie. Berlin, Germany: Springer.

Maercker, A., Mohiyeddini, C., Muller, M., Xie, W., Hui Yang, Z., Wang, J., \& Muller, J. (2009). Traditional versus modern values, self-perceived interpersonal factors, and posttraumatic stress in Chinese and German crime victims. Psychology and Psychotherapy: Theory, Research and Practice, 82(2), 219-232. https://doi.org/10.1348/147608308X380769

Maniglio, R. (2009). The impact of child sexual abuse on health: A systematic review of reviews. Clinical Psychology Review, 29(7), 647-657. https://doi.org/10.1016/j.cpr.2009.08.003

Marinova, Z., Maercker, A., Küffer, A., Robinson, M. D., Wojdacz, T. K., Walitza, S., . . Burri, A. (2017). DNA methylation profiles of elderly individuals subjected to indentured childhood labor and trauma. BMC Medical Genetics, 18(1), Article 21. https://doi.org/10.1186/s12881-017-0370-2

Meili, I., Heim, E., \& Maercker, A. (2018). Culturally shared metaphors expand contemporary concepts of resilience and post-traumatic growth: Contrasting an indigenous Brazilian community and a Swiss rural community. Medical Humanities. Advance online publication. https://doi.org/10.1136/medhum-2018-011450

Nemeroff, C. B. (2016). Paradise lost: The neurobiological and clinical consequences of child abuse and neglect. Neuron, 89(5), 892-909. https://doi.org/10.1016/j.neuron.2016.01.019

Norman, R. E., Byambaa, M., De, R., Butchart, A., Scott, J., \& Vos, T. (2012). The long-term health consequences of child physical abuse, emotional abuse, and neglect: A systematic review and meta-analysis. PLoS Medicine, 9(11), Article e1001349.

https://doi.org/10.1371/journal.pmed.1001349

Pape, J. C., \& Binder, E. B. (2014). Psychotrauma als Risiko für spätere psychische Störungen:

Epigenetische Mechanismen [Psychological trauma as risk for delayed psychiatric disorders: epigenetic mechanisms]. Nervenarzt, 85(11), 1382-1389.

https://doi.org/10.1007/s00115-014-4085-8 
Perkonigg, A., Lorenz, L., \& Maercker, A. (2018). Prevalence and correlates of ICD-11 adjustment disorder: Findings from the Zurich Adjustment Disorder Study. International fournal of Clinical and Health Psychology, 18(3), 209-217. https://doi.org/10.1016/j.ijchp.2018.05.001

Pielmaier, L., \& Maercker, A. (2011). Psychological adaptation to life-threatening injury in dyads: The role of dysfunctional disclosure of trauma. European fournal of Psychotraumatology, 2(1), Article 8749. https://doi.org/10.3402/ejpt.v2i0.8749

Rechsteiner, K., Maercker, A., \& Tol, V. (2019). "It should not have happened": Idioms and metaphorical expressions related to trauma in an indigenous community in India. Manuscript submitted for publication.

Rosen, G. M., Spitzer, R. L., \& McHugh, P. R. (2008). Problems with the post-traumatic stress disorder diagnosis and its future in DSM V. British fournal of Psychiatry, 192(1), 3-4. https://doi.org/10.1192/bjp.bp.107.043083

Rosner, R., Pfoh, G., Rojas, R., Brandstätter, M., Rossi, A., Lumbeck, G., . . Geissner, E. (2014). Anhaltendende Trauerstörung: Manuale für die Einzel- und Gruppentherapie Göttingen, Germany: Hogrefe.

Schauer, M., Neuner, F., \& Elbert, T. (2011). Narrative exposure therapy: A short-term treatment for traumatic stress disorder (2nd ed.). Cambridge, MA, USA: Hogrefe Publishing.

Schnyder, U., \& Cloitre, M. (2015). Evidence based treatments for trauma-related psychological disorders: A practical guide for clinicians. Heidelberg, Germany: Springer.

Silove, D., Ventevogel, P., \& Rees, S. (2017). The contemporary refugee crisis: An overview of mental health challenges. World Psychiatry: Official fournal of the World Psychiatric Association (WPA), 16(2), 130-139. https://doi.org/10.1002/wps.20438

Somasundaram, D. (2014). Addressing collective trauma: Conceptualisations and interventions. Intervention, 12(Suppl. 1), 43-60. https://doi.org/10.1097/WTF.0000000000000068

Spröber, N., Schneider, T., Rassenhofer, M., Seitz, A., Liebhardt, H., König, L., \& Fegert, J. M. (2014). Child sexual abuse in religiously affiliated and secular institutions: A retrospective descriptive analysis of data provided by victims in a government-sponsored reappraisal program in Germany. BMC Public Health, 14, Article 282. https://doi.org/10.1186/1471-2458-14-282

Stein, D. J., McLaughlin, K. A., Koenen, K. C., Atwoli, L., Friedman, M. J., Hill, E. D., . . Kessler, R. C. (2014). DSM-5 and ICD-11 definitions of posttraumatic stress disorder: Investigating "narrow" and "broad" approaches. Depression and Anxiety, 31(6), 494-505. https://doi.org/10.1002/da.22279

Teicher, M. H., \& Samson, J. A. (2013). Childhood maltreatment and psychopathology: A case for ecophenotypic variants as clinically and neurobiologically distinct subtypes. American fournal of Psychiatry, 170(10), 1114-1133. https://doi.org/10.1176/appi.ajp.2013.12070957

Teicher, M. H., \& Samson, J. A. (2016). Annual Research Review: Enduring neurobiological effects of childhood abuse and neglect. fournal of Child Psychology and Psychiatry, 57(3), 241-266. https://doi.org/10.1111/jcpp.12507 
Teicher, M. H., Samson, J. A., Polcari, A., \& McGreenery, C. E. (2006). Sticks, stones, and hurtful words: Relative effects of various forms of childhood maltreatment. American fournal of Psychiatry, 163(6), 993-1000. https://doi.org/10.1176/ajp.2006.163.6.993

Thompson, C. T., Vidgen, A., \& Roberts, N. P. (2018). Psychological interventions for post-traumatic stress disorder in refugees and asylum seekers: A systematic review and meta-analysis. Clinical Psychology Review, 63, 66-79. https://doi.org/10.1016/j.cpr.2018.06.006

Tol, W. A., Barbui, C., Galappatti, A., Silove, D., Betancourt, T. S., Souza, R., . . van Ommeren, M. (2011). Mental health and psychosocial support in humanitarian settings: Linking practice and research. Lancet, 378(9802), 1581-1591. https://doi.org/10.1016/S0140-6736(11)61094-5

Trickett, P. K., Noll, J. G., Susman, E. J., Shenk, C. E., \& Putnam, F. W. (2010). Attenuation of cortisol across development for victims of sexual abuse. Development and Psychopathology, 22(1), 165-175. https://doi.org/10.1017/S0954579409990332

Turecki, G., \& Meaney, M. J. (2016). Effects of the social environment and stress on glucocorticoid receptor gene methylation: A systematic review. Biological Psychiatry, 79(2), 87-96. https://doi.org/10.1016/j.biopsych.2014.11.022

Viola, T. W., Salum, G. A., Kluwe-Schiavon, B., Sanvicente-Vieira, B., Levandowski, M. L., \& GrassiOliveira, R. (2016). The influence of geographical and economic factors in estimates of childhood abuse and neglect using the Childhood Trauma Questionnaire: A worldwide metaregression analysis. Child Abuse \& Neglect, 51, 1-11. https://doi.org/10.1016/j.chiabu.2015.11.019

Von Lersner, U., \& Kizilhan, J. I. (2017). Kultursensitive Psychotherapie [Cultural sensitive psychotherapy]. Göttingen, Germany: Hogrefe.

Whealin, J. M., Yoneda, A. C., Nelson, D., Hilmes, T. S., Kawasaki, M. M., \& Yan, O. H. (2017). A culturally adapted family intervention for rural Pacific Island veterans with PTSD. Psychological Services, 14(3), 295-306. https://doi.org/10.1037/ser0000186

World Health Organization. (2016). Problem management plus: Individual psychological help for adults impaired by distress in communities exposed to adversity. Geneva, Switzerland: WHO.

World Health Organization. (2018). ICD-11 for Mortality and Morbidity Statistics. Retrieved from https://icd.who.int/browse11/1-m/en

Yehuda, R., Daskalakis, N. P., Bierer, L. M., Bader, H. N., Klengel, T., Holsboer, F., \& Binder, E. B. (2016). Holocaust exposure induced intergenerational effects on FKBP5 methylation. Biological Psychiatry, 80(5), 372-380. https://doi.org/10.1016/j.biopsych.2015.08.005 